\title{
BacStalk: A comprehensive and interactive image analysis software tool for bacterial cell biology
}

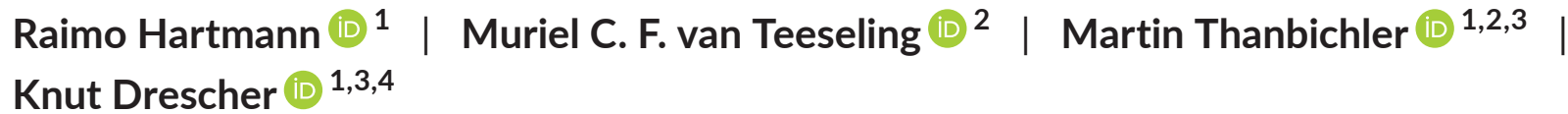

${ }^{1}$ Max Planck Institute for Terrestrial Microbiology, Marburg, Germany

${ }^{2}$ Department of Biology, Philipps-Universität Marburg, Germany

${ }^{3}$ LOEWE Center for Synthetic Microbiology, Marburg, Germany

${ }^{4}$ Department of Physics, Philipps-Universität Marburg, Germany

\section{Correspondence}

Knut Drescher, Max Planck Institute for Terrestrial Microbiology, Marburg, Germany Email: k.drescher@mpi-marburg.mpg.de

Funding information

Max-Planck-Gesellschaft; European

Molecular Biology Organization, Grant/

Award Number: ALTF1396-2015; H2O20

European Research Council, Grant/

Award Number: 716734; Deutsche

Forschungsgemeinschaft, Grant/Award

Number: 192445154 - SFB987; Human

Frontier Science Program, Grant/Award

Number: CDA00084/2015-C

\begin{abstract}
Prokaryotic cells display a striking subcellular organization. Studies of the underlying mechanisms in different species have greatly enhanced our understanding of the morphological and physiological adaptation of bacteria to different environmental niches. The image analysis software tool BacStalk is designed to extract comprehensive quantitative information from the images of morphologically complex bacteria with stalks, flagella, or other appendages. The resulting data can be visualized in interactive demographs, kymographs, cell lineage plots, and scatter plots to enable fast and thorough data analysis and representation. Notably, BacStalk can generate demographs and kymographs that display fluorescence signals within the two-dimensional cellular outlines, to accurately represent their subcellular location. Beyond organisms with visible appendages, BacStalk is also suitable for established, nonstalked model organisms with common or uncommon cell shapes. BacStalk, therefore, contributes to the advancement of prokaryotic cell biology and physiology, as it widens the spectrum of easily accessible model organisms and enables highly intuitive and interactive data analysis and visualization.
\end{abstract}

\section{KEYWORDS}

Bacterial cell biology, image analysis, software tool

\section{1 | INTRODUCTION}

The study of prokaryotic model species has greatly contributed to the advancement of cell biology. Originally viewed as simple organisms lacking internal structure, prokaryotes are now acknowledged to display an impressive degree of subcellular organization (Shapiro et al., 2009). A central question in cell biology is how cells spatiotemporally regulate the positioning and activities of (macro)molecular complexes in order to grow, divide, and adequately respond to changes in their extracellular environment. Studies in established prokaryotic model organisms, such as Bacillus subtilis, Escherichia coli, Caulobacter crescentus, and Vibrio cholerae, have yielded important insights into the mechanisms that organize prokaryotic cells. However, these species only represent a small part of the highly diverse prokaryotic world. Therefore, it is not surprising that the analysis of less commonly studied model species has recently uncovered a series of novel cell-biological pathways (Eswara and Ramamurthi, 2017; Caccamo and Brun, 2018). These findings have significantly

Raimo Hartmann and Muriel C.F. van Teeseling contributed equally.

This is an open access article under the terms of the Creative Commons Attribution-NonCommercial License, which permits use, distribution and reproduction in any medium, provided the original work is properly cited and is not used for commercial purposes.

(c) 2020 The Authors. Molecular Microbiology published by John Wiley \& Sons Ltd 
improved our understanding of prokaryotic cell biology and revealed that prokaryotes have often evolved multiple solutions to the same regulatory problem (Laloux and Jacobs-Wagner, 2014; TreunerLange and Søgaard-Andersen, 2014).

Although bacterial cell biology has primarily been studied in species that grow symmetrically and divide by binary fission, there are various other ways of proliferation among bacteria, for which the underlying mechanisms are still largely unknown (Angert, 2005). A treasure trove for the analysis of cell growth and division are the Alphaproteobacteria, a group of species characterized by an enormous diversity of cell shapes, growth modes, physiological properties and life cycles (Randich and Brun, 2015). Alphaproteobacteria occur in a wide range of environments, such as in the rhizosphere (e.g., Rhizobiales), in oceanic (e.g., Hyphomonas species) and freshwater (e.g., Hyphomicrobium and Caulobacter species) habitats, and inside eukaryotic cells (e.g., Rickettsiales). Many of them, including the well-established model organism $C$. crescentus, form one or multiple extrusions of the cell envelope (termed prosthecae or stalks), whose length often depends on nutrient availability. Alphaproteobacteria can grow through longitudinal growth, polar growth, or budding and divide by symmetric or asymmetric binary fission (Staley, 1968; Brown et al., 2012; Randich and Brun, 2015). Another, largely unexplored means of reproduction in this class is stalk-terminal budding, a process in which new offspring is formed by expansion and subsequent separation of the terminal stalk segment (Hirsch, 1974; Cserti et al., 2017). This growth mode is observed for various species and appears to have arisen at least twice during evolution in the freshwater Hyphomicrobium/Rhodomicrobium and the marine Hyphomonas/Hirschia lineages, respectively (Cserti et al., 2017). It involves a complex developmental program influenced by nutrient availability, including the establishment of several distinct growth zones, the highly asymmetric placement of division machinery to the bud neck (Cserti et al., 2017), and a novel mode of chromosome segregation (Jung et al., 2019). Importantly, apart from their complex morphologies, stalked bacteria are highly polarized and undergo cell cycle-dependent changes in their shape and physiology (Poindexter, 1964; Pate et al., 1973; Whittenbury and Dow, 1977; Moore, 1981; Williams et al., 2016; Caccamo and Brun, 2018). Their study is thus bound to provide important new insights into the mechanisms that spatiotemporally organize bacterial cells (Wagner and Brun, 2007). Moreover, it will help to unveil the molecular principles underlying the evolution of morphological traits in bacteria (Jiang et al., 2014) and clarify how bacterial morphology depends on environmental conditions.

Primary characteristics of a suitable model organism for prokaryotic cell biology are: (a) ease of cultivation, (b) amenability to genetic manipulation, and (c) the ability to extract quantitative phenotypic information via light microscopy, aided by efficient automated image analysis. Although a variety of bacterial species are easy to cultivate and genetically amenable, the capabilities of available image analysis software are still limiting. The development of dedicated software tools, such as MicrobeTracker (Sliusarenko et al., 2011), Oufti (Paintdakhi et al., 2016), MicrobeJ (Ducret et al.,
2016), BactMAP (van Raaphorst et al., 2019), and others (Guberman et al., 2008; van Raaphorst et al., 2017; Goñi-Moreno et al., 2017), has facilitated the automated analysis of light microscopy images of common prokaryotic model organisms and now even permits impressive high-throughput screenings of mutant libraries based on the morphology and intracellular organization (Campos et al., 2018). Although these software packages are becoming more versatile, they lack the possibility to extract quantitative information for prokaryotes with special morphologies. In the case of stalked bacteria, for instance, the detection of stalks in microscopy images routinely still occurs manually.

Existing image analysis algorithms cannot reliably recognize stalks because these thin structures are characterized by a very low contrast in phase-contrast images, making automated segmentation challenging. When, as a work-around, stalks are fluorescently labeled to facilitate their visualization, their dimensions are typically increased significantly by diffraction. Although the latest version of MicrobeJ can relate filamentous structures to corresponding cells if they are fluorescently labeled and segmentable by thresholding (Ducret et al., 2016), the possibilities for analyzing stalked bacteria are very limited. At present, it is for instance not possible to visualize concatenated fluorescence profiles along cells and stalks. In addition, the image analysis of stalked budding bacteria remains very difficult as the mother cell, stalk, and bud cannot be detected as connected entities that make up a single cell.

To provide a powerful image analysis software for prokaryotes with complex cell morphologies, such as stalked bacteria, we developed BacStalk, a MatLab-based software tool for automated, label-free, time-resolved image analysis of stalked and non-stalked bacteria.

Using an intuitive graphical user interface, BacStalk enables the detection of fluorescence patterns as well as the morphometric analysis of cell bodies, and, if present, their corresponding stalks and buds. Importantly, BacStalk can visualize fluorescence signals in (populations of) cells in interactive demographs and kymographs in which the cells can be aligned according to relevant morphological features and cell morphologies are visualized in addition to the fluorescence signals. BacStalk, therefore, enables both in-depth cell biological studies as well as quantitative analyses of (fluorescently labeled) environmental samples, of fluorescent reporter strains for bacterial physiology (Kremer et al., 2019) and of environmentally induced morphological changes. Here, we describe and discuss the capabilities and functionality of BacStalk, based on image data sets of different bacterial species.

\section{RESULTS \& DISCUSSION}

BacStalk is designed for label-free bacterial cell and stalk detection in phase-contrast images at pixel accuracy. As stalks usually have very low contrast and are hardly visible, conventional thresholding approaches fail to separate them reliably from the image background. BacStalk overcomes these difficulties by implementing a two-step 
approach: First, cells are identified by enhancing image features of a typical length scale by applying bandpass filtering, followed by automatic thresholding. In the second step, BacStalk detects connected stalks by performing local morphological operations. In detail, a shell is constructed around each cell, consisting of all pixels surrounding the cell that are positioned at a user-defined distance away from the cell. The intensity values of all pixels of this shell are then scanned for subtle intensity differences (either in the phase-contrast or brightfield image or in any desired fluorescence channel) that could indicate a potential stalk attachment point (Figure 1a). For each pixel of this shell, the intensity is compared to the mean intensity of all pixels of this shell, by computing the $z$-score (i.e., the number of standard deviations that an intensity value deviates from the mean shell intensity). If the lowest $z$-score in phase-contrast images (or highest $z$-score in fluorescence images) exceeds a user-defined threshold value, the pixel with this $z$-score is defined as the stalk attachment point. The stalk backbone is then generated through repeated dilation starting from the stalk attachment point, by looking for other pixels in a user-defined range that are again below (or above, for fluorescence) a defined $z$-score (Figure 1a). Thus, if present, the stalk is constructed from the stalk attachment point outwards in a directional manner. If another cell is encountered during stalk propagation, both the initial cell and the touched counterpart are defined as related and can be treated as a connected structure (mother cell and bud) during cell tracking and the downstream analysis. The larger cell is, hereby, defined as a mother cell. In cells and buds of stalked budding bacteria, the cell polarity is clearly defined by the stalk attachment point and indicated by a yellow dot at the end of the cell's

(a) Stalk detection

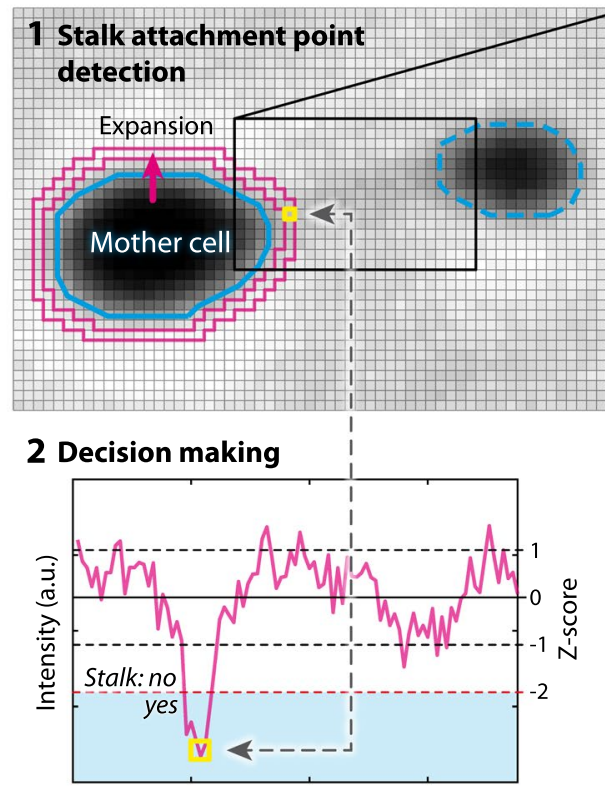

Shell pixel index

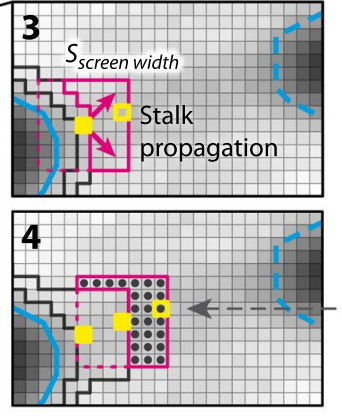

3-6 Tracing intensity minimum

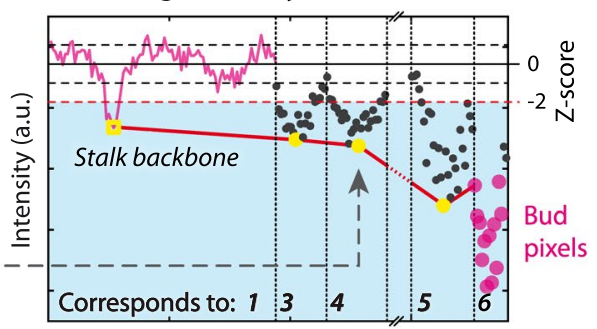

Stalk propagation pixel index
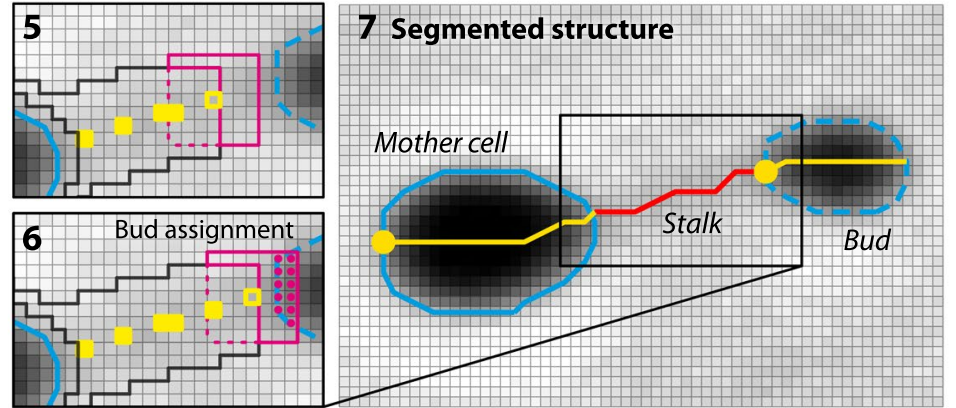

(b) Demo-/kymograph cell alignment options

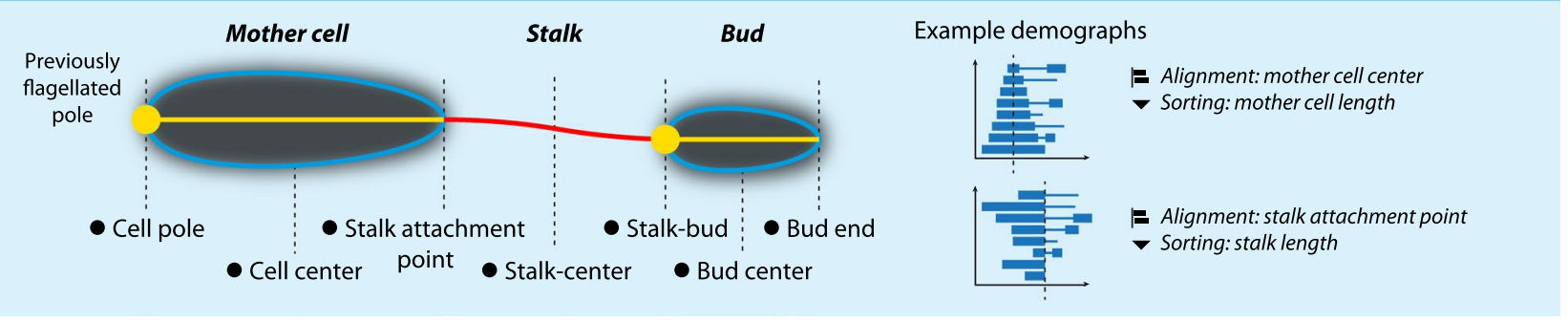

FIGURE 1 BacStalk detects stalks in phase-contrast images by tracing their subtle intensity patterns. Mother cells, stalks, and buds are treated as connected morphological entities that can be appropriately aligned and sorted during quantitative data visualization. (a) Stalk detection algorithm. Steps 1-2: The stalk attachment point is determined by expanding each cell and identifying the pixel of lowest intensity (yellow pixel) inside the expanded cell-shell (magenta), whose intensity is below a typical z-score (number of standard deviations away from the mean). Steps 3-6: The stalk backbone is constructed by dilating the stalk attachment point by the stalk screening width and finding the next pixel of intensity below a typical z-score in the newly expanded area (magenta). The dilation is repeated for each valid backbone pixel and stopped if the stalk becomes too faint or another cell is encountered. Step 7: If the encountered cell is smaller than the initial cell, it is classified as a bud to the larger mother cell. If it is larger, the relation is inverted. (b) For visualization in demo-/kymographs, the cells can be aligned to all relevant positions (marked here with a solid black dot) and sorted according to any of the extracted parameters 
medial axis (Figure 1a). In the case of stalk-free swarmer cells, the identity of the cell pole is guessed based on the cell morphology or the existence of intensity features, but it can also be interactively changed manually at any stage of the analysis. As unambiguous stalk assignment in clumps of multiple cells is typically impossible, cell clumps are automatically recognized and excluded from further analysis. The approach described above is relatively insensitive to uneven background illumination in the microscopy images and does not require any background correction.

As an alternative to BacStalk's inbuilt cell detection algorithm, BacStalk can also import the segmentation masks from other software packages, enabling the use of BacStalk's analysis features for images with custom segmentation requirements. BacStalk's cell and stalk detection process are relatively fast, requiring approximately 4.5 s per image (for 40 cells in a $2048 \times 2048$ pixel image using a computer with an Intel i7-6700K processor and 16 GB memory). On computers with multiple CPU cores, BacStalk can parallelize computing tasks to reduce processing time and to facilitate high-throughput analysis. Batch analyses of images can benefit from the possibility to differentiate between different strains or growth conditions using the custom metadata option during the import of images, thus allowing the analysis of multiple strains at the same time using the same settings.

To test the functionality of BacStalk, we first compared the results of its cell segmentation algorithm to that of MicrobeJ (Ducret et al., 2016) and Oufti (Paintdakhi et al., 2016) by quantifying the cellular dimensions of 105 C. crescentus cells as detected by each of these software packages (Supporting Information Table S1). This analysis showed some segmentation algorithm-specific differences in the cell length and width values but very similar standard deviations.

We then tested BacStalk on several species of stalked bacteria to verify the robustness of the stalk detection algorithm. The algorithm performed well for $C$. crescentus (Figure 2a), Brevundimonas aveniformis (Figure 2b), and $\mathrm{H}$. neptunium (Figures 3 and 4). To examine the accuracy of the stalk detection algorithm, we used a $C$. crescentus strain in which the terminal segments of the stalk are fluorescently labeled by a GFP-tagged version of the stalk-specific protein StpX (Hughes et al., 2010). When correlating the stalks identified by BacStalk based on phase-contrast images in the presence of StpX-GFP signals (Supporting Information Figure S1), we observed that stalks were correctly identified for $91 \%$ of the cells ( $n=616$ ) without modifying the default image segmentation settings, indicating that the stalk detection algorithm works robustly. Most of the misidentifications occurred for cells that were slightly out of focus (only cells that were considerably out of focus had been excluded from the analysis manually) or in cases where the stalk was very small, clearly below the 5 pixels defined as the default minimum stalk length. To investigate whether stalks of different lengths are detected reliably, we imaged C. crescentus cells grown in the presence or absence of phosphate, as it has previously been shown that $C$. crescentus cells strongly elongate their stalk upon phosphate starvation (Schmidt and Stanier, 1966). The analysis of 500 cells grown in these two conditions verified that stalks of cells grown with phosphate were indeed considerably shorter and less variable in length $(1.47 \pm 0.75 \mu \mathrm{m})$ than stalks of cells deprived of phosphate $(6.45 \pm 2.90 \mu \mathrm{m})$ (Figure 2a). Interestingly, we found that in the latter condition the cell length correlated at least partially with the stalk length. Stalk detection also works in very low contrast conditions in which the stalk is hard to distinguish from the background by eye, as generally observed for B. aveniformis (Figure 2b). For this species, BacStalk identifies $60 \pm 5 \%$ of all cells as stalked ( $n=750$, from five independent experiments), which is comparable to the stalked population fraction measurement of $46 \pm 4 \%$ reported by a previous study (Curtis, 2017). Furthermore, our analysis showed that cells with stalks display on average slightly longer cell bodies $(2.47 \pm 0.56 \mu \mathrm{m})$ than cells without stalks $(2.05 \pm 0.45 \mu \mathrm{m})$.

In addition to stalks, the stalk detection algorithm can also identify other single polar cell appendages, such as flagella (Figure 2c). Since these structures are often not visible in phase-contrast or bright-field images, the algorithm can be applied to stained appendages visualized by fluorescence microscopy. BacStalk was able to reliably detect flagella of Shewanella putrefaciens cells that were labeled with a fluorescent dye (Figure 2c) (Kühn et al., 2017). This analysis showed that the flagellar lengths vary considerably between cells (from 0.33 to $6.88 \mu \mathrm{m}$, with an average of $3.35 \pm 1.87 \mu \mathrm{m}$ ).

In case of the stalked budding bacterium $H$. neptunium, BacStalk can accurately distinguish between mother cells, stalks, and buds for the wild type as well as for mutants with altered morphology (Figure 3). Thus, BacStalk quantifies phenotypes in a more accurate, detailed, and faster manner than the current standard in the field (i.e., quantification via manual measurements). The power of such automated quantifications is illustrated by the fact that the morphological phenotype of an $\mathrm{H}$. neptunium $\Delta$ pbp1x mutant (i.e., an increased length of budding cells), which was described qualitatively before (Cserti et al., 2017), can now be precisely quantified (Figure 3): the combined length of mother cells and stalks was $3.2 \pm 1.3 \mu \mathrm{m}$ long for $\Delta p b p 1 x$ cells and $2.7 \pm 0.7 \mu \mathrm{m}$ for wild-type cells (both $n=183$ ). The quantitative analysis by BacStalk also provided additional new insights. For instance, BacStalk revealed that the distribution of stalk lengths is dramatically broadened in the $\Delta p b p 1 x$ background and that the bud area correlates partially with the total length of the concatenated entity of mother cell, stalk, and bud.

The combination of features offered by BacStalk also facilitates detailed analyses of protein localization experiments based on fluorescence signals. To this end, BacStalk can detect the brightest focus inside cells after applying a 3-by-3 mean filter to the cell. The distance to the pole of this brightest fluorescence spot is then measured along the medial axis. By default, no fluorescence normalization or background subtraction is performed during the analysis, yet the user may choose whether to subtract the background or normalize fluorescence during the data visualization. To visualize protein localization, BacStalk can display combined intensity profiles of the mother cell, stalk, and bud, which can be aligned according to relevant cellular landmark locations and sorted by any measured property of the combined structure (Figure 1b). To generate intensity 


\section{(a) C. crescentus cells}

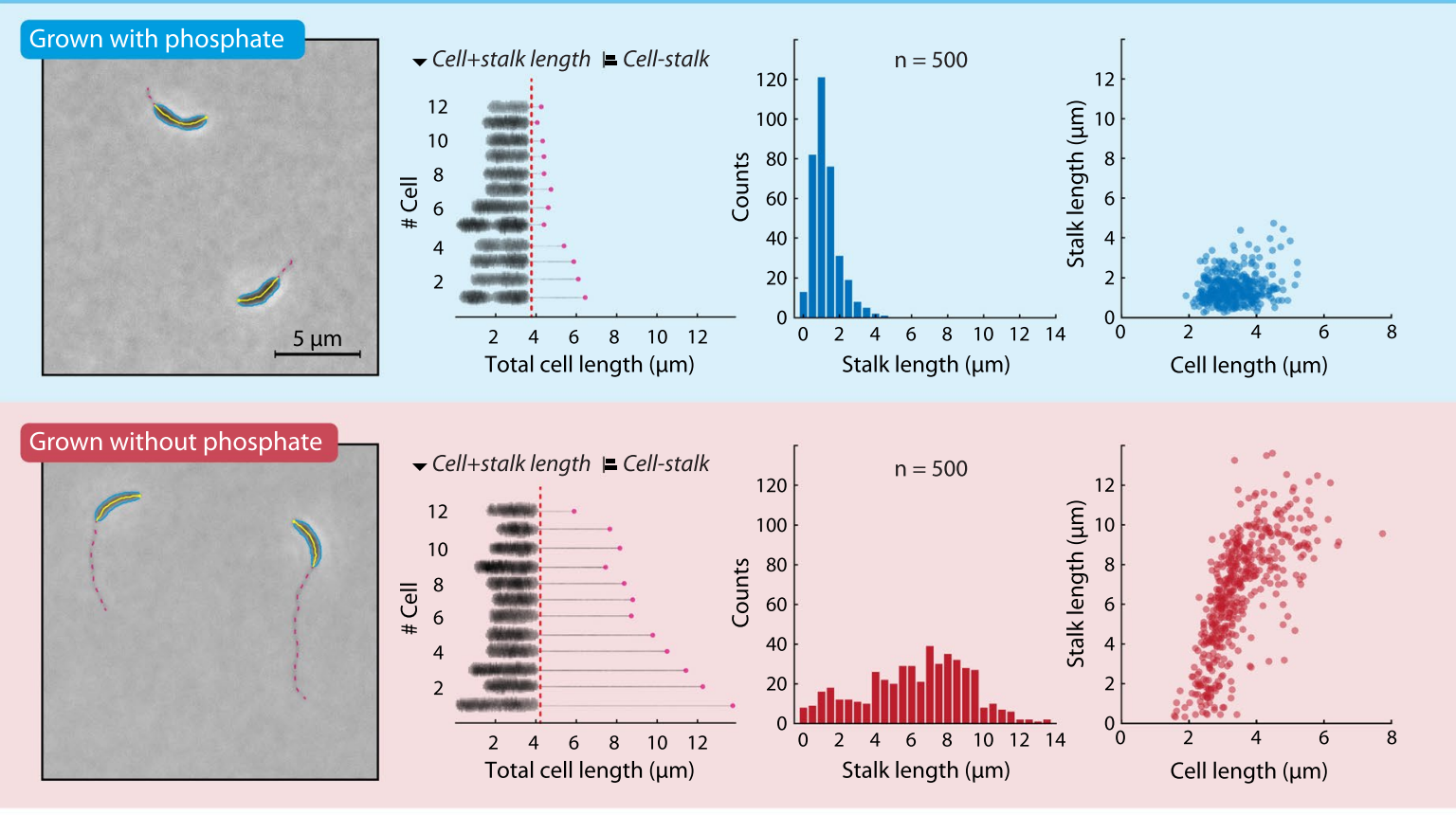

(b) B. aveniformis cells

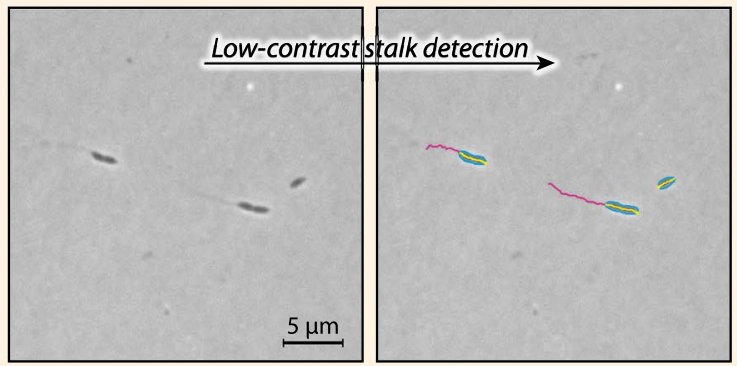

Length of cell bodies of $B$. aveniformis cells $(n=150)$

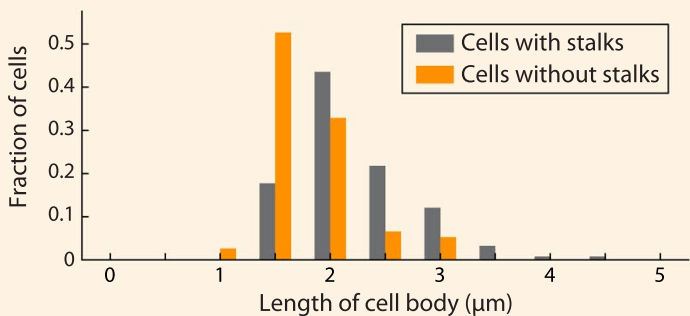

(c) S. putrefaciens cells
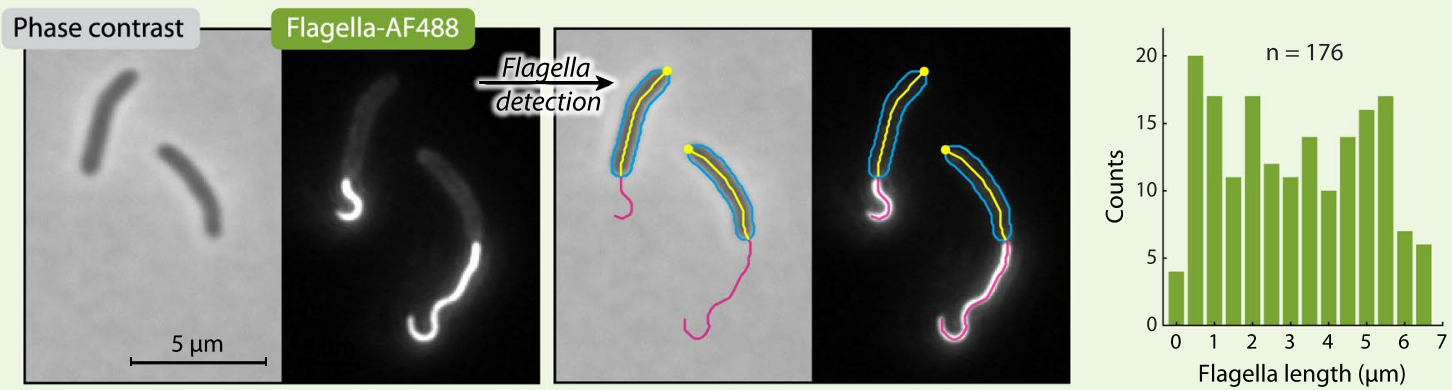

FIGURE 2 BacStalk detects cell bodies, stalks or other polar appendages, and buds in multiple species of stalked bacteria. (a) C. crescentus cells with normal and elongated stalks are detected and characterized separately. Screenshots of the output generated by BacStalk show cell outlines in light blue, medial axes in yellow, and stalks as magenta lines. Two-dimensional demographs display 12 representative cells for both conditions, aligned at the stalk attachment point and sorted according to the cell + stalk length after straightening of the cells by medial axis coordinate system transformations. The distributions of stalk lengths are displayed in histograms. In addition, the correlations between the lengths of the cell bodies and the corresponding stalks are visualized via scatterplots. (b) The stalk detection algorithm also recognizes stalks that are hardly visible by eye, as demonstrated on B. aveniformis cells. Screenshots of the output generated by BacStalk are as described in panel A. The lengths of cells with and without stalks are displayed in a histogram. (c) Other polar appendages can also be detected by the stalk detection algorithm, using image data obtained in the fluorescence channel. In this case, polar flagella of S. putrefaciens (strain S4401) were stained using a fluorescent thiol-reactive maleimide dye that binds covalently to exposed cysteine residues in the major flagellin protein. The fluorescence signal was detected in the GFP channel. Screenshots were created as described in panel a. The lengths of flagella are displayed in a histogram 

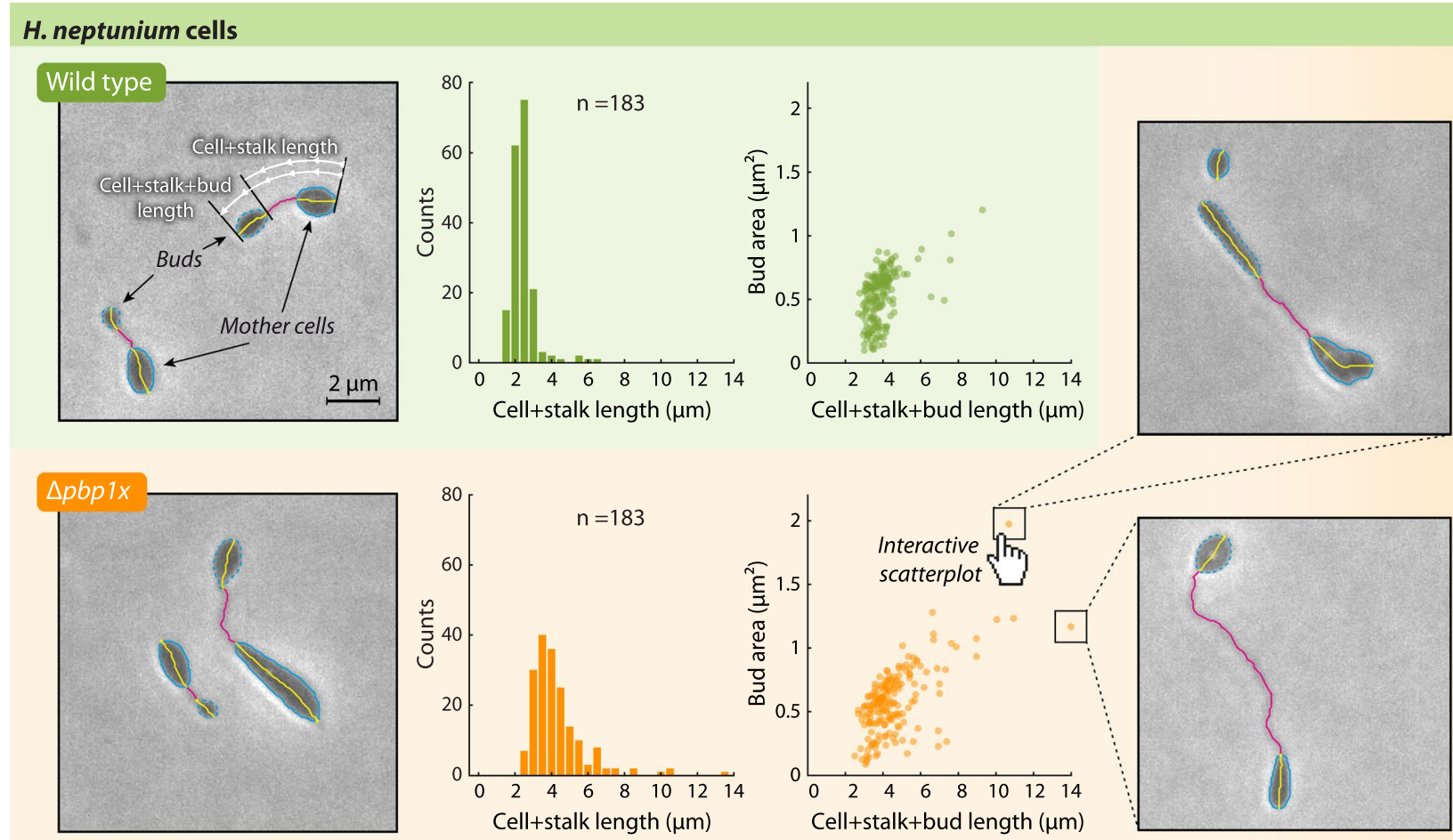

FIGURE 3 BacStalk can be used for the analysis of mutants that differ in cell shape. Morphological differences between $H$. neptunium wild-type and $\Delta p b p 1 x$ cells are detected by quantitative analysis. In screenshots of the output generated by BacStalk, cell outlines are indicated by light blue lines, with bud cells highlighted by dashed outlines and medial axes in yellow. Upon deletion of the pbp $1 x$ gene, the cell + stalk length increases and shows a broader distribution, as shown in histograms. In addition, the population contains more cells with a larger bud area, which correlates with an increased total cell (cell + stalk+bud) length, as demonstrated by scatter plots comparing the bud area to the total cell length. The scatter plots generated by BacStalk are interactive: the underlying cell image is displayed and highlighted in the user interface, when clicking on a particular data point, for example, in the scatter plot

profiles along the medial axis of a cell, BacStalk fits a mesh of evenly spaced lines into each cell perpendicular to its medial axis, similar to Oufti (Paintdakhi et al., 2016). For each point on the medial axis, the mean or maximum of the intensity values along the corresponding line is calculated to obtain smooth intensity profiles along the cell medial axis. In addition, the mesh is used to perform a medial axis coordinate system transformation in order to reorient curved cells such that they can be arranged next to each other in two-dimensional (2D) demographs and kymographs to conserve the full spatial information of patterns inside the cells (Figures 2a, 4b,c, 5b). These $2 \mathrm{D}$ demo- and kymographs intuitively visualize the imaging data and provide important additional information in cases where the fluorescent protein of interest is located away from the medial axis. Furthermore, the fluorescence intensity profiles can be normalized and background-corrected per cellular entity.

BacStalk provides a very flexible data visualization environment for protein localization experiments. The 1D and 2D demo- and kymographs, the use of concatenated intensity profiles, the possibility to align these profiles based on the morphologically relevant criteria (e.g., alignment at the junction between the mother cell and stalk, or flagellum, or at a specific pole), and the option to display specific subsets of cells (i.e., swarmer cells, stalked cells without buds, and budding cells) are instrumental in understanding the localization behavior of proteins in different cell types in a mixed population. This is exemplified by an analysis of the localization dynamics of the histidine kinase CckA in $H$. neptunium using BacStalk, which verified the cell cycle-dependent localization previously observed in a qualitative manner (Leicht et al., 2020). BacStalk provides a means to automatically plot the intracellular localization of CckA-Venus in demographs separately for swarmer cells, stalked cells without buds, and budding cells (Figure 4a). All demographs were sorted according to the length of the cellular structures: the first demograph is sorted by the length of the cell, the second by the combined lengths of the mother cell and stalk, and the third by the combined lengths of the mother cell, stalk, and bud. Cells were aligned at the cell center, the cell-stalk junction, or the bud pole opposite to the stalk, respectively.

Apart from its powerful visualization tools, BacStalk includes special features to track individual cells in time-lapse experiments and to analyze dynamic protein localization during stalk-terminal budding. In time-lapse experiments, cells that display an overlap in position in consecutive frames are identified as the same cell and the lineage information of cells is saved. As an example, we reinvestigated the cell cycle-dependent localization of the guanylate cyclase PleD in $\mathrm{H}$. neptunium cells (Figure 4b). The analysis by BacStalk provides a detailed quantification of the cell type-specific localization pattern that has previously only been described in a qualitative manner (Jung et al., 2015). In swarmer cells, PleD-Venus localizes at the flagellated pole of the mother cell. In stalked cells, the PleD-Venus 
(a) Localization of CckA-Venus in H. neptunium

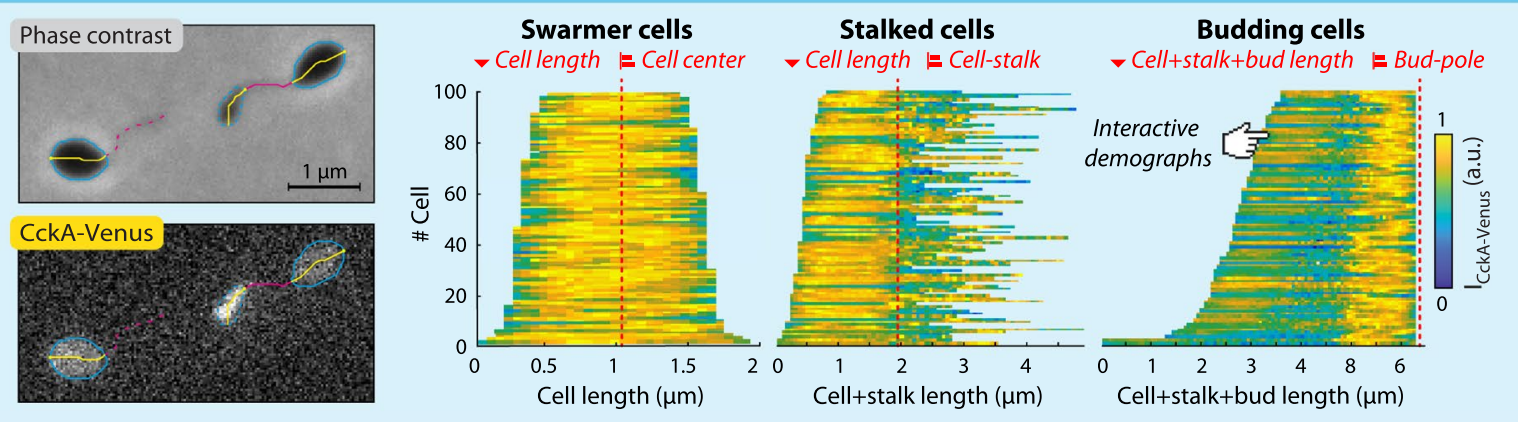

(b) Dynamic localization of PleD-Venus in H. neptunium

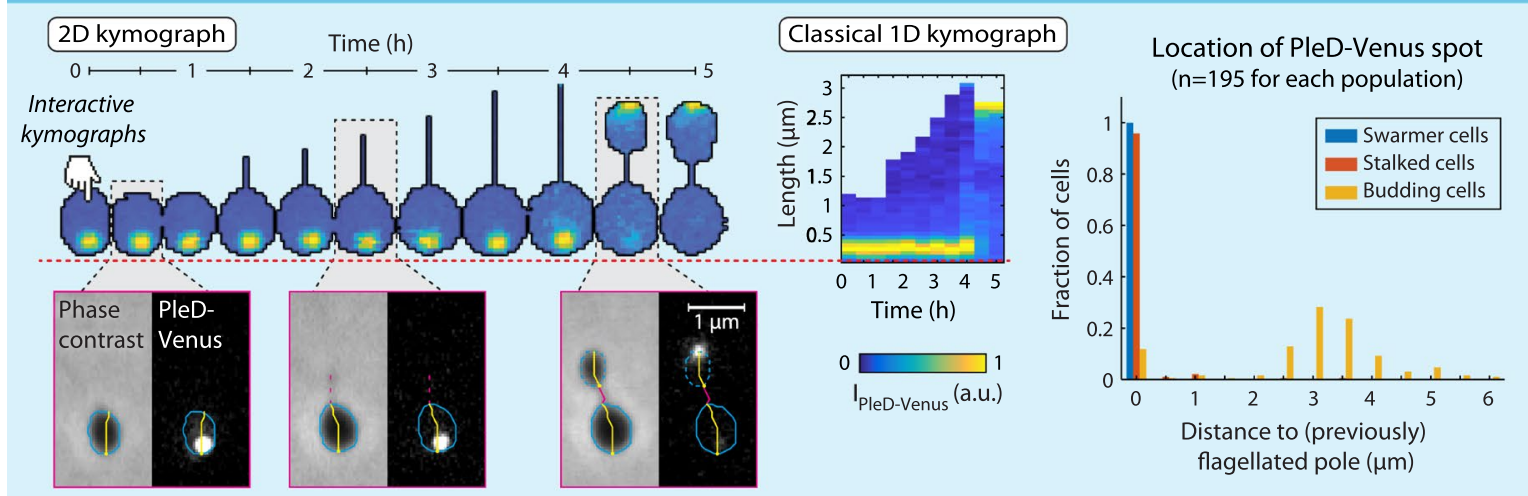

(c) Multi-color localization dynamics of DnaN-Venus and ParB-Cerulean in H. neptunium

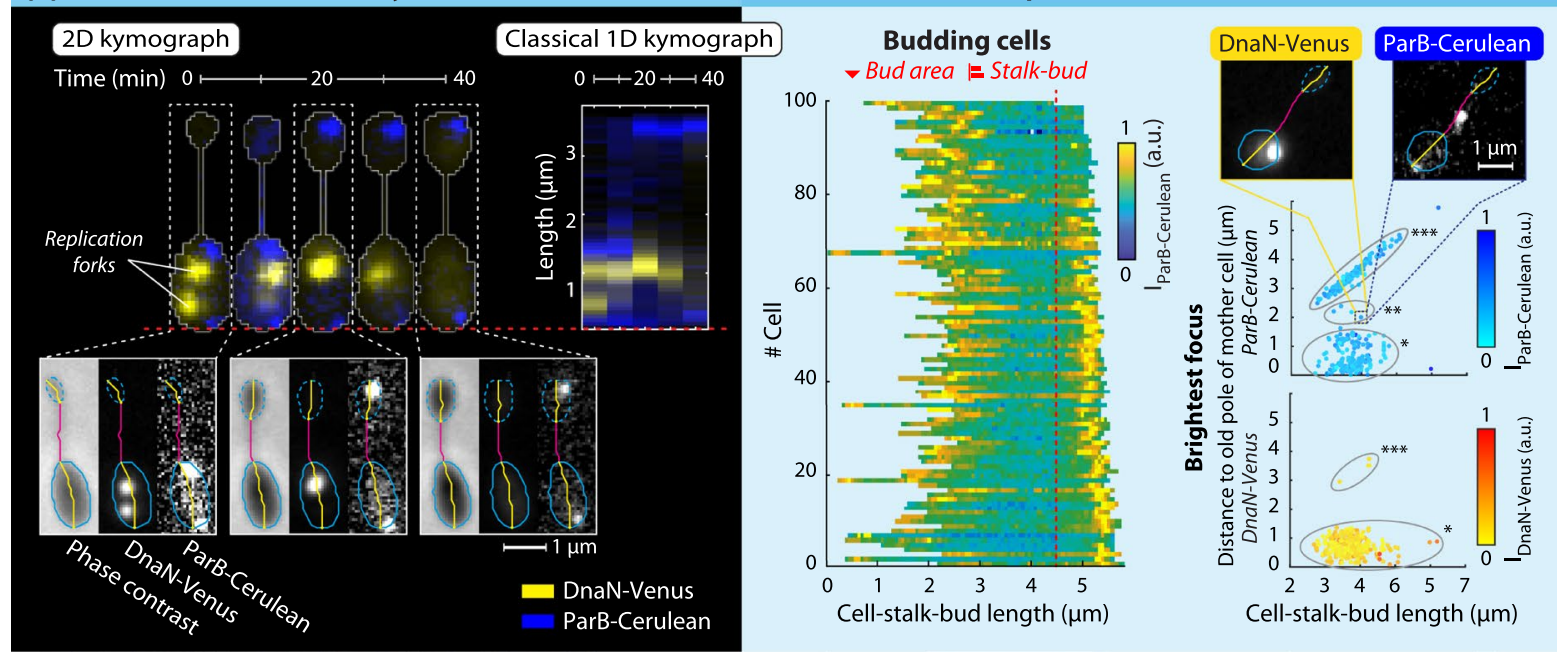

FIGURE 4 BacStalk enables the comprehensive and intuitive visualization of complex fluorescence patterns. (a) Analysis of the subcellular localization of CckA-Venus in H. neptunium cells at different stages of development. Cells are aligned and ordered according to biologically relevant parameters (see red text above each demograph). The fluorescence profiles represent the mean fluorescence intensities along the medial axis after background subtraction and normalization such that the maximum fluorescence of each cell is equal. (b) Dynamic localization of PleD-Venus in H. neptunium, displayed as a 2D-kymograph (left) and a classical 1D-kymograph (middle) after background subtraction and normalization of the maximum fluorescence signal per time point. The histogram (right) shows the distance of the pixel with maximum PleD-Venus intensity from the old pole of the mother cell for swarmer cells, stalked cells, and budding cells. (c) The relative dynamics of multiple fluorescence signals in H. neptunium are visualized by 2D and 1D (classical) kymographs. The 2D kymograph clearly reveals DNA transfer to the bud (illustrated by the movement of the blue ParB-Cerulean signal) before the entire DNA replication process is completed (as indicated by the delocalization of the yellow DnaN-Venus signal). The fluorescence of ParB-Cerulean was corrected for the background and normalized (as the Cerulean signal bleached quickly), whereas the signal of DnaN-Venus was not background-corrected (in order to illustrate the difference between the association of DnaN-Venus with the chromosome and its later dissociation). Demograph: Cells are aligned by the stalk-bud connection point and sorted according to the bud area (see red text above the demograph), revealing that ParB-Cerulean localization to the bud requires a minimum bud area. Scatter plots: ParB-Cerulean is present either in the mother cell $\left({ }^{*}\right)$ or at the flagellated pole of the bud $\left(^{* * *}\right)$ and only in rare cases in the stalk $\left({ }^{* *}\right)$. In contrast, the highest fluorescent signal location of DnaN-Venus is predominantly present in mother cells $\left({ }^{*}\right)$. Dots in the scatterplot are colored relative to the fluorescence intensity of the brightest spot, showing that in the rare cases where the brightest spot of DnaN-Venus fluorescence is present in the bud (***), the DnaN-Venus signal was low (and thus replication was not ongoing) 


\section{(a) E. coli cells}

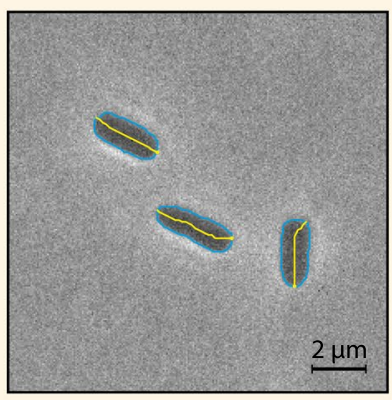

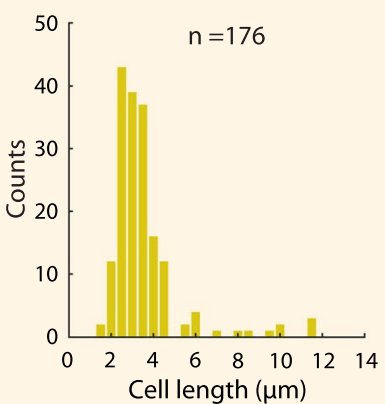

(b) M. xanthus cells

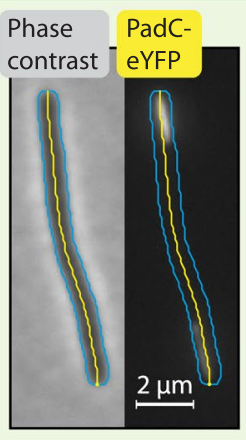

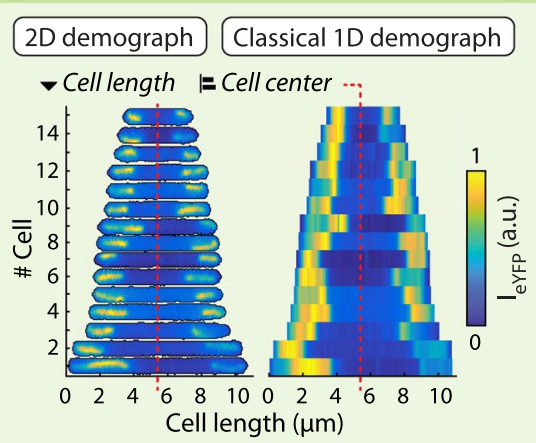

FIGURE 5 BacStalk is also a powerful tool for the analysis of non-stalked bacteria. (a) Images and histograms showing lengths of E. coli TOP10 cells. (b) Images and 1D/2D demographs of M. xanthus cells (strain MO039), demonstrating the localization of PadC-eYFP to the subpolar regions

focus also tends to be located at the flagellated pole of the mother cell, whereas in most budding cells it is detected in the bud at the pole opposite the stalk. Although the relocation of PleD from the old pole of the mother cell to the old pole of the daughter cell can be traced in a 1D kymograph, the 2D kymograph additionally facilitates the correlation of protein relocation with cell morphogenesis (e.g., bud formation). Quantification of the localization of PleD-Venus in different cell types is also possible by determining and plotting its distance from the old pole of the mother cell (Figure $4 b$ ).

To investigate the patterns of multiple fluorescence signals at different wavelengths simultaneously, BacStalk can create multi-channel kymographs (or demographs): the kymograph in Figure $4 \mathrm{c}$ shows $H$. neptunium cells undergoing replication, in which (a) the replisome component DnaN is tagged with the fluorescent protein Venus and (b) the origin of replication is followed with the help of a ParB-Cerulean fusion, which binds parS sites near the chromosomal origin of replication (Jung et al., 2019). This two-color approach corroborates the relative timing of replisome movement and origin segregation revealed previously by manual analysis (Jung et al., 2019), and verifies that the origin of replication already moves to the bud before the replication is completed (as visualized by the delocalization of DnaN-Venus). In addition, the 2D kymograph visualization clearly identifies both replication forks as separate entities (distinct DnaN foci inside the first cell in kymograph, Figure 4c). As in Figure 4b, we determined the distance of the ParB-Cerulean and DnaN-Venus foci to the old pole of the mother cell. Using this representation, Figure $4 \mathrm{c}$ confirms that the replication origin (tagged by ParB-Cerulean) is only transferred to the bud once a certain bud size has been reached and that the process of origin movement through the stalk must be fast, as the origin was captured inside the stalk in only $\sim 1 \%$ of all cells that were analyzed ( 4 out of 378 cells).

BacStalk provides several analysis tools that greatly simplify data exploration and visualization. Customizable cell measurements can be added by users and are listed, together with all other measured parameters, in an interface that allows for filtering and the identification of specific sub-populations of interest. The measured data for each cell can be exported to several standard formats. Similar to MicrobeJ (Ducret et al., 2016), all plots created with BacStalk are interactive: clicking on a data point in a scatter plot (Figures 2-4) or on a fluorescence profile in a demo- or kymograph (Figures 4 and 5) displays the underlying cell, so that the corresponding raw image data and phenotype can be assessed. Furthermore, the output images of BacStalk showing the results of analyses or images of cells are publication-ready: all images in Figures $2-5$ have only been minimally edited after their export from BacStalk (e.g., by changing the background color, cropping or minor editing of the axes). BacStalk offers the possibility to obtain all underlying raw data, so that they can be used for downstream analyses with other software tools, as described in the BacStalk online documentation.

The features of BacStalk that are exemplified above for stalked and flagellated bacteria, most notably its interactivity, its ease of use (see Figure 6 for a description of the workflow), the one-click generation of 1D and 2D kymo- and demographs, and the ability to output publication-ready editable images, are also applicable to the investigation of classical, non-stalked model organisms, such as E. coli and M. xanthus (Figure 5). The generation of interactive 2D demographs is a feature that is currently not available in any other image analysis software package. Its usefulness is demonstrated by an analysis of the localization dynamics of YFP-tagged PadC, an adapter protein connecting the chromosome partitioning ATPase ParA to subpolarly located bactofilin polymers in M. xanthus (Lin et al., 2017). Here, the $2 \mathrm{D}$ representation provides important information about the spatial arrangement of the filaments within the cell that cannot be appreciated in standard 1D demographs (Figure 5B). It should be noted that for stalked and non-stalked species, images should only have a low to moderate cell density, as no cell-splitting functionality is implemented in the current version of BacStalk.

BacStalk was written in MatLab to make use of its built-in figure customization and editing capabilities for generating publication-ready vector graphics, and to provide advanced users with easy access to the underlying processed data. However, our main goal in the design of the software was to make it as user-friendly 


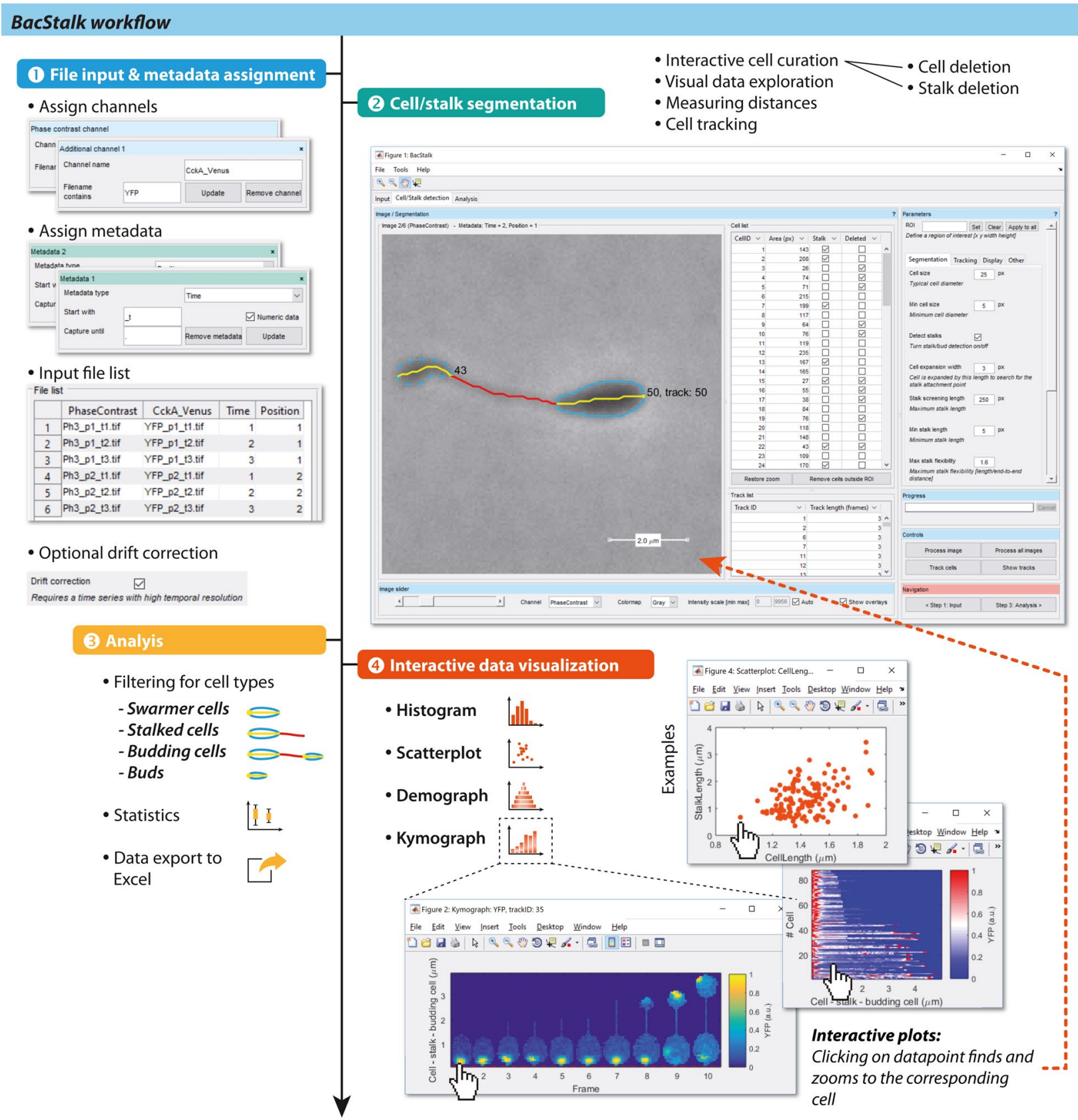

FIGURE 6 Typical workflow in BacStalk. Step 1: Channel and metadata information are extracted from file names based on keywords. For time-resolved image series, sample drift correction (image registration) can be performed. Step 2: BacStalk provides a powerful customizable image viewer for visual data exploration, cell and stalk segmentation, curation, and tracking. Image processing can be optionally sped up by using MatLab's Parallel Computing Toolbox. Step 3: The analysis tab provides tools for creating subsets of the bacterial population, calculating statistical parameters, and exporting the data to separate spreadsheet applications such as Microsoft Excel. Step 4: With a single click, complex data visualizations can be created, which are interactive and easily customizable using MatLab's built-in plot editor to generate publication-ready vector graphics figures

as possible and applicable on first-try without any programming knowledge. This ease of use is achieved by BacStalk's powerful and fast graphical user interface (Figure 6). In addition, the user is supported by a comprehensive documentation and detailed video tutorials, which are available online at https://drescherlab.org/ data/BacStalk together with the open source code and a standalone pre-compiled version that does not require a MatLab license.
Overall, BacStalk facilitates high-throughput, in-depth, single-cell image analysis of stalked and non-stalked bacteria. It thus enables the study of many interesting and environmentally relevant bacteria as novel model organisms, provides tools for more detailed analyses of established model organisms, and therefore, constitutes an indispensable tool for bacterial cell biology and physiology. 


\section{3 | EXPERIMENTAL PROCEDURES}

\section{1 | Bacterial strains}

All strains analyzed in this study are listed in Supporting Information Table S2. The plasmids and oligonucleotides used for their construction are listed in Supporting Information Tables S3 and S4.

\subsection{Cultivation of cells}

C. crescentus wild-type cells were pre-grown (at $28^{\circ} \mathrm{C}$ ) in peptoneyeast-extract (PYE) medium (Poindexter, 1964), subsequently diluted 1:20 into M2-glucose (M2G) minimal medium (Ely, 1991) with phosphate or without phosphate $\left(\mathrm{M}^{2} \mathrm{G}^{-\mathrm{P}}\right)$ to induce stalk elongation, and cultivated for another $24 \mathrm{hr}$ prior to analysis. C. crescentus strain NR5064 (stpX-gfp) was grown in PYE medium at $28^{\circ} \mathrm{C}$. B. aveniformis was grown in the PYE medium at $28^{\circ} \mathrm{C}$ and $\mathrm{H}$. neptunium in MB medium (Difco Marine Broth 2216, BD Biosciences, USA) at $28^{\circ} \mathrm{C}$. E. coli and S. putrefaciens were grown in LB medium (at $37^{\circ} \mathrm{C}$ and $28^{\circ} \mathrm{C}$, respectively). M. xanthus cells were grown at $32^{\circ} \mathrm{C}$ in CTT medium (Hodgkin and Kaiser, 1977). All incubations were performed with shaking at $210 \mathrm{rpm}$. The synthesis of PadC-eYFP was induced for $3 \mathrm{hr}$ with $5 \mu \mathrm{M}$ vanillate. For imaging, cells were grown to early exponential phase $\left(\mathrm{OD}_{600}=0.2-0.45\right)$ and spotted on pads consisting of $1 \%$ agarose (peqGOLD Universal Agarose, peqlab, Germany) and the respective medium. In the case of $H$. neptunium strain JR47, the cells were imaged on pads prepared with 4-fold diluted MB medium, whereas M. xanthus M0039 cells were applied to pads made with 5 -fold diluted CTT, in both cases to decrease background fluorescence. S. putrefaciens S4401 cells were first treated as described previously (Kühn et al., 2017) with the thiol-reactive dye Alexa Fluor $488 C_{5}$ maleimide (Thermo Fisher Scientific) to stain the flagella and imaged on pads made of LM100 minimal medium (Kühn et al., 2017).

\section{3 | Light microscopy}

All strains, except for E. coli, S. putrefaciens, and the H. neptunium wild-type and $\Delta p b p 1 X$ strains, were analyzed with a Zeiss Axio Observer.Z1 inverted microscope (Zeiss, Germany) equipped with a Zeiss Plan-Apochromat 100x/1.4 oil immersion Ph3 objective, Chroma ET-YFP and ET-CFP filter sets, and a pco.edge $4.2 \mathrm{mHQ}$ camera (PCO, Germany). S. putrefaciens cells were imaged with a Zeiss Axio Imager.M1 microscope equipped with a Zeiss Plan-Apochromat 100x/1.4 oil immersion Ph3 objective, a Chroma ET-GFP filter set, and a pco.edge $4.2 \mathrm{mHQ}$ camera (PCO, Germany). E. coli and H. neptunium wild-type and $\Delta p b p 1 \mathrm{X}$ cells were imaged with a Nikon Ti-E inverted epifluorescence microscope equipped with a Nikon PlanApochromat $\lambda 100 x / 1.45$ oil immersion Ph3 objective and an Andor Zyla 4.2plus sCMOS camera.

\section{ACKNOWLEDGMENTS}

We are grateful to Oliver Leicht, Emöke Cserti, Alexandra Jung, Lin Lin, Manuel Osorio Valeriano, and Julia Rosum for the construction of plasmids and strains used in this study. We would like to thank Marco Kühn and Kai Thormann for providing S. putrefaciens strain S4401 and Patrick Viollier for the C. crescentus StpX-GFP strain. We are grateful to Revathi L. Pulpetta for imaging strain JR47 and Manuel Osorio Valeriano for imaging M. xanthus cells. In addition, we would like to thank Adrian Izquierdo Martinez, Maria Billini, Manuel Osorio Valeriano, Revathi L. Pulpetta and Till, as well as the attendants of the BacStalk workshops in Marburg for their feedback after testing BacStalk prior to release. Sanika Vaidya, Konstanin Neuhaus, and Miriam Bayer helped with the growth and staining of S. putrefaciens and Eric Jelli helped with the code and website. This work was supported by grants from the Germany Research Foundation (Project 192445154-Collaborative Research Center SFB 987; to M.T. and K.D.), the Max Planck Society (to M.T. and K.D.), the Human Frontier Science Program (CDA00084/2015-C; to K.D.), and the European Research Council (StG-716734; to K.D.). M.C.F.v.T. acknowledges support by an EMBO Long-Term Fellowship (ALTF13962015), which was co-funded by the European Commission through the Marie-Skłodowska Curie Actions program (LTFCOFUND2013, GA-2013-609409).

\section{CONFLICT OF INTEREST}

The authors declare no conflict of interest.

\section{DATA AVAILABILITY STATEMENT}

Software source code and a pre-compiled version that does not require a MatLab license, as well as full documentation and video tutorials are available at https://drescherlab.org/data/BacStalk. Image data and processed data used in this study are available from the corresponding author upon reasonable request.

\section{ORCID}

Raimo Hartmann (iD https://orcid.org/0000-0002-4924-6402

Muriel C. F. van Teeseling (iD https://orcid.

org/0000-0002-8297-2481

Martin Thanbichler iD https://orcid.org/0000-0002-1303-1442

Knut Drescher (D) https://orcid.org/0000-0002-7340-2444

\section{REFERENCES}

Angert, E.R. (2005) Alternatives to binary fission in bacteria. Nature Reviews Microbiology, 3, 214-224.

Brown, P.J.B., de Pedro, M.A., Kysela, D.T., Henst, C.V., Van der Henst, C., Kim, J., De Bolle, X., Fuqua, C. and Brun, Y. V. (2012) Polar growth in the alphaproteobacterial order Rhizobiales. Proceedings of the National Academy of Sciences of the United States of America, 109, 1697-1701.

Caccamo, P.D. and Brun, Y.V. (2018) The molecular basis of noncanonical bacterial morphology. Trends in Microbiology, 26, 191-208.

Campos, M., Govers, S.K., Irnov, I., Dobihal, G.S., Cornet, F. and JacobsWagner, C. (2018) Genome-wide phenotypic analysis of growth, cell morphogenesis, and cell cycle events in Escherichia coli. Molecular Systems Biology, 14, e7573. 
Cserti, E., Rosskopf, S., Chang, Y.-W., Eisheuer, S., Selter, L., Shi, J., Regh, C., Koert, U., Jensen, G.J. and Thanbichler, M. (2017) Dynamics of the peptidoglycan biosynthetic machinery in the stalked budding bacterium Hyphomonas neptunium. Molecular Microbiology, 103, 875-895.

Curtis, P.D. (2017) Stalk formation of Brevundimonas and how it compares to Caulobacter crescentus. PLOS ONE, 12, e0184063.

Ducret, A., Quardokus, E.M. and Brun, Y.V. (2016) MicrobeJ, a tool for high throughput bacterial cell detection and quantitative analysis. Nature Microbiology, 1, 1-7.

Ely, B. (1991) Genetics of Caulobacter crescentus. Methods in Enzymology, 204, 372-384.

Eswara, P.J. and Ramamurthi, K.S. (2017) Bacterial cell division: nonmodels poised to take the spotlight. Annual Review of Microbiology, 71, 393-411.

Goñi-Moreno, Á., Kim, J. and de Lorenzo, V. (2017) Cell Shape: A user-friendly image analysis tool for quantitative visualization of bacterial cell factories inside. Biotechnology Journal, 12, 1600323.

Guberman, J.M., Fay, A., Dworkin, J., Wingreen, N.S. and Gitai, Z. (2008) PSICIC: noise and asymmetry in bacterial division revealed by computational image analysis at sub-pixel resolution. PLoS Computational Biology, 4, e1000233.

Hirsch, P. (1974) Budding bacteria. Annual Review of Microbiology, 28 391-440.

Hodgkin, J. and Kaiser, D. (1977) Cell-to-cell stimulation of movement in nonmotile mutants of Myxococcus. Proceedings of the National Academy of Sciences of the United States of America, 74, 2938-2942.

Hughes, H.V., Huitema, E., Pritchard, S., Keiler, K.C., Brun, Y.V. and Viollier, P.H. (2010) Protein localization and dynamics within a bacterial organelle. Proceedings of the National Academy of Sciences of the United States of America, 107, 5599-5604.

Jiang, C., Brown, P.J.B., Ducret, A. and Brun, Y.V. (2014) Sequential evolution of bacterial morphology by co-option of a developmental regulator. Nature, 506, 489-493.

Jung, A., Eisheuer, S., Cserti, E., Leicht, O., Strobel, W., Möll, A., Schlimpert, S., Kühn, J. and Thanbichler, M. (2015) Molecular toolbox for genetic manipulation of the stalked budding bacterium Hyphomonas neptunium. Applied and Environment Microbiology, 81, 736-744.

Jung, A., Raßbach, A., Pulpetta, R.L., van Teeseling, M.C.F., Heinrich, K., Sobetzko, P., Serrania, J., Becker, A. and Thanbichler, M. (2019) Two-step chromosome segregation in the stalked budding bacterium Hyphomonas neptunium. Nature Communications, 10, 3290.

Kremer, K., van Teeseling, M.C.F., Schada von Borzyskowski, L., Bernhardsgrütter, I., van Spanning, R.J.M., Gates, A.J., RemusEmsermann, M.N.P., Thanbichler, M. and Erb, T.J. (2019) Dynamic metabolic rewiring enables efficient acetyl coenzyme $A$ assimilation in Paracoccus denitrificans. mBio, 10, e00805-e819.

Kühn, M.J., Schmidt, F.K., Eckhardt, B. and Thormann, K.M. (2017) Bacteria exploit a polymorphic instability of the flagellar filament to escape from traps. Proceedings of the National Academy of Sciences, 114, 6340-6345.

Laloux, G. and Jacobs-Wagner, C. (2014) How do bacteria localize proteins to the cell pole? Journal of Cell Science, 127, 11-19.

Leicht, O., van Teeseling, M. C. F., Panis, G., Reif, C., Wendt, H., Viollier, P. H. and Thanbichler, M. (2020) Integrative and quantitative view of the CtrA regulatory network in a stalked budding bacterium. PLOS Genetics. https://doi.org/10.1371/journal.pgen.1008724

Lin, L., Osorio Valeriano, M., Harms, A., Søgaard-Andersen, L., and Thanbichler, M. (2017) Bactofilin-mediated organization of the ParABS chromosome segregation system in Myxococcus xanthus. Nature Communications, 8, 1817.
Moore, R.L. (1981) Hyphomicrobium and other bacteria. Annual Review of Microbiology, 35, 567-585.

Paintdakhi, A., Parry, B., Campos, M., Irnov, I., Elf, J., Surovtsev, I. and Jacobs-Wagner, C. (2016) Oufti: an integrated software package for high-accuracy, high-throughput quantitative microscopy analysis. Molecular Microbiology, 99, 767-777.

Pate, J.L., Porter, J.S. and Jordan, T.L. (1973) Asticcacaulis biprosthecum sp.nov. Life cycle, morphology and cultural characteristics. Antonie van Leeuwenhoek, 39, 569-583.

Poindexter, J.S. (1964) Biological properties and classification of the Caulobacter group. Bacteriological Reviews, 28, 231-295.

Randich, A.M. and Brun, Y.V. (2015) Molecular mechanisms for the evolution of bacterial morphologies and growth modes. Frontiers in Microbiology, 6, 580.

Schmidt, J. M. and Stanier, R. Y. (1966). The development of cellular stalks in bacteria. Journal of Cell Biology, 28, 423-436.

Shapiro, L., McAdams, H.H. and Losick, R. (2009) Why and how bacteria localize proteins. Science, 326, 1225-1228.

Sliusarenko, O., Heinritz, J., Emonet, T. and Jacobs-Wagner, C. (2011) High-throughput, subpixel precision analysis of bacterial morphogenesis and intracellular spatio-temporal dynamics. Molecular Microbiology, 80, 612-627.

Staley, J.T. (1968) Prosthecomicrobium and Ancalomicrobium: new prosthecate freshwater bacteria. Journal of Bacteriology, 95, 1921-1942.

Treuner-Lange, A. and Søgaard-Andersen, L. (2014) Regulation of cell polarity in bacteria. Journal of Cell Biology, 206, 7-17.

van Raaphorst, R., Kjos, M. and Veening, J.-W. (2017) Chromosome segregation drives division site selection in Streptococcus pneumoniae. Proceedings of the National Academy of Sciences of the United States of America, 114, E5959-E5968.

van Raaphorst, R., Kjos, M. and Veening, J.-W. (2019) BactMAP: an R package for integrating, analyzing and visualizing bacterial microscopy data. Molecular Microbiology, 113, 297-308.

Wagner, J.K. and Brun, Y.V. (2007) Out on a limb: how the Caulobacter stalk can boost the study of bacterial cell shape. Molecular Microbiology, 64, 28-33.

Whittenbury, R. and Dow, C.S. (1977) Morphogenesis and differentiation in Rhodomicrobium vannielii and other budding and prosthecate bacteria. Bacteriological Reviews, 41, 754-808.

Williams, M., Hoffman, M.D., Daniel, J.J., Madren, S.M., Dhroso, A. Korkin, D., Givan, S.A., Jacobson, S.C. and Brown, P.J.B. (2016) Shortstalked Prosthecomicrobium hirschii cells have a Caulobacter-like cell cycle. Journal of Bacteriology, 198, 1149-1159.

\section{SUPPORTING INFORMATION}

Additional Supporting Information may be found online in the Supporting Information section.

How to cite this article: Hartmann R, van Teeseling MCF, Thanbichler M, Drescher K. BacStalk: A comprehensive and interactive image analysis software tool for bacterial cell biology. Mol Microbiol. 2020;114:140-150. https://doi. org/10.1111/mmi.14501 